Marathonlauf

\section{Fördert er die Arterienverkalkung?}

Wer regelmäßig an Marathons teilnimmt und für die Läufe über Jahre hinweg intensiv trainiert, tut damit seinem Herzen nicht nur Gutes . Ausgerechnet eine Gruppe von 25 besonders fitten Veteranen des Langstreckenlaufs, die an 25 und mehr Läufen teilgenommen hatten, wiesen stärker verkalkte Koronarien auf als eine gleichaltrige, lauffaule Vergleichsgruppe. Und das, obwohl Herzfrequenz, Gewicht und Body-Mass-In$\operatorname{dex}(B M I)$ bei den Läufern deutlich niedriger lagen (wobei Letzterem allerdings immer weniger prädiktives Potenzial beigemessen wird; s. nebenstehende Meldung).

\section{Die Studienleiter:}

\section{selbst passionierte Läufer}

Der Clou an der Sache: Die Studie wurde initiiert von zwei Medizinern, die selbst leidenschaftlich Marathon laufen, Vater und Sohn Robert und Jonathan Schwartz, tätig am Minneapolis Heart Institute, resp. an der Universität Colorado, Denver. Statt sich durch die Ergebnisse in ihrer Laufleidenschaft bestätigt zu sehen, mussten sie eine herbe Überraschung hinnehmen: Das Volumen verkalkter Plaques, gemessen mithilfe der CT-Angiografie, maß bei den Läufern $274 \mathrm{~mm}^{3}$, bei den Kontrollen dagegen nur $169 \mathrm{~mm}^{3}$.

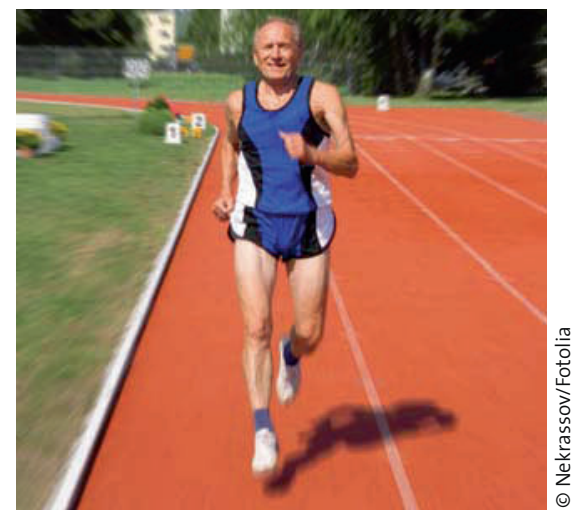

Nicht so gesund, wie er denkt.

Bei den Ursachen musste das Forscherteam passen. Jonathan Schwartz führte erhöhte Herzraten und Blutdrücke während des Trainings als mögliche Erklärung an und spekulierte weiter, dass evtl. auch der anhaltende anaerobe Zustand während des Langstreckenlaufs zu antioxidanzienbedingten Schäden führen könnte. Diese Zusammenhänge müssen noch untersucht werden.

EOII

Schwartz JG, et al. ACC 2010 Scientific Sessions; 16.3.2010, Atlanta, USA

\title{
SCHLAGANFALL
}

\section{Prävention edelbitter}

Süßigkeiten fallen eigentlich nicht gerade in die Rubrik gesunde Ernährung. Doch das Naschen von dunkler, flavonoidreicher Schokolade scheint (auch) positive gesundheitliche Auswirkungen zu haben. In zwei von drei prospektiven Kohortenstudien zeigte sich ein geringeres Risiko für Schlaganfälle sowie ein Rückgang der Schlaganfallmortalität. Es kann allerdings nicht ausgeschlossen werden, dass andere gesunde Verhaltensweisen von Schokoladeessern zu dem guten Ergebnis beigetragen haben. Ungeklärt ist auch die Frage nach der optimalen Dosis: Die nicht unerheblichen Mengen an Fett und Zucker könnten den mutmaßlichen Nutzen der Flavonoide konterkarieren.

Sahib S et al. American Academy of Neurology Meeting 2010, online 17.2.2010

\section{ERHÖHTES INFARKTRISIKO \\ Gicht gefährdet \\ auch Frauenherzen}

Frauen, die an Gicht leiden, haben ein deutlich erhöhtes Herzinfarktrisiko. In einer siebenjährigen Beobachtungsstudie lag es um $40 \%$ höher als das von Frauen ohne Gicht. Bei Männern ist ein solcher Zusammenhang bereits aus früheren Studien bekannt. Es wird vermutet, dass infolge der Hyperurikämie die Proliferation und Entzündung der glatten Gefäßmuskulatur sowie die Plättchenaggregationsfähigkeit zunehmen.

Fazit für die Praxis: Wenn bei einem Patienten eine Gicht besteht, müssen kardiovaskuläre Risikofaktoren besonders aggressiv angegangen werden.

\section{KHK-Risikofaktoren}

\section{Niedriger IO fast so schlimm wie rauchen}

Personen mit einem niedrigen Intelligenzquotienten haben ein erhöhtes Risiko für Herz-Kreislauf-Erkrankungen und eine erhöhte kardiovaskuläre Mortalität. Mit Daten der West-of-Scotland-Twenty-07Studie (1145 Probanden, die über 20 Jahre beobachtet wurden) konnte jetzt eine Rangfolge von neun unterschiedlichen Risikofaktoren erstellt werden.

Demnach beträgt der relative Risikoindex für Zigarettenrauchen 5,58, für einen niedrigen IQ_3,20, für niedriges Einkommen 2,61 , für einen hohen systolischen Blutdruck 2,61 und für Bewegungsmangel 2,06. Je höher der Wert, desto bedeutender ist der Risikofaktor.

Für den hohen Stellenwert des niedrigen IO sind zwei Erklärungen denkbar: Zum einen könnten Personen mit einem niedrigen IO besonders zu einem ungesunden Lebensstil neigen. Denkbar ist aber auch, dass häufige Erkrankungen und suboptimale Ernährung für den niedrigen IO und das erhöhte kardiovaskuläre Risiko verantwortlich sind.

RM I

Europ J Cardiovasc Prev Rehab 2010;17(1):24
Ann Rheum Dis 2010; DOI:10.1136/ ard.20091227701

\section{RELIGIOSITÄT UND GESUNDHEIT}

\section{Glaube schützt nicht vor Herzerkrankungen}

Die Frage, ob Glaube gesund macht, wurde jetzt nach wissenschaftlicher Überprüfung verneint. Anhand einer 4-Jahres-Studie mit 5500 Teilnehmern hatten Ärzte den Zusammenhang zwischen Religiosität und kardiovaskulärem Risiko analysiert. Studienteilnehmer, die viel beteten oder meditierten, hatten ebenso häufig subklinische oder manifeste kardiovaskuläre Erkrankungen wie nicht religiöse Probanden. Sehr gläubige Menschen rauchten zwar weniger, waren dafür aber deutlich häufiger adipös. 